\title{
Preparation and Evaluation of 5-Fluorouracil Loaded Microsponges for Treatment of Colon Cancer
}

\author{
Mahmoud H Othman ${ }^{1 *}$, Gamal M Zayed ${ }^{1}$, Gamal H El-Sokkary ${ }^{2}$, Usama F Ali ${ }^{3}$ and Ahmed AH Abdellatif \\ ${ }^{1}$ Department of Pharmaceutics and Industrial Pharmacy, Faculty of Pharmacy, Al-Azhar University, Assiut, Egypt \\ ${ }^{2}$ Deparment of Zoology, Faculty of Science, Assiut University, Assiut, Egypt \\ ${ }^{3}$ Department of Pharmaceutics, Faculty of Pharmacy, Minia University, Minia, Egypt
}

\begin{abstract}
5-Fluorouracil (5-FU) has a wide anticancer activity versus several types of solid tumors. The activity of 5-FU can be improved and its toxicity can be diminished by enhancing the relative specific accumulation in the tumor regions. The aim of this work was to develop Eudragit RS100 based 5-FU microsponges (MS) for treatment of colon cancer. Oil in oil emulsion solvent diffusion method was used for the preparation of 5-FU sustained release Eudragit RS100 MS. MS were characterized for their encapsulation efficiency, production yield, drug polymer interaction and drug release profiles. Shape, surface morphology visualized by scanning electron microscope (SEM) and particle size of MS was investigated using laser light scattering technique and. Eventually, HCT 116 and CACO2 cell lines were used for determination of cell viability by MTT assay. The results showed that all prepared MS were spherical in shape with several pores on their surfaces. The production yield was in $(62.76 \% \pm 1.06 \%$ and $93.80 \% \pm 1.75 \%)$, encapsulation efficiency was in $(71.80 \% \pm 1.62 \%$ and $101.3 \% \pm 2.60 \%)$ and particle size was in ( $53.11 \mu \mathrm{m} \pm 41.03$ $\mathrm{nm}$ and $118.12 \mu \mathrm{m} \pm 48.21 \mathrm{~nm}$ ). Fourier transform infrared revealed that there is no chemical interaction between 5-FU and Eudragit RS100. MS loaded 5-FU was more effective than 5-FU itself as shown by cell viability assay. The results demonstrated that 5 -FU with Eudragit RS100 was successfully formulated as sustained release manner and could be a substitution delivery method of 5-FU for oral anticancer treatment.
\end{abstract}

Keywords: 5-Fluorouracil; Microsponges; Eudragit RS100; Cell viability; HCT116 and CACO-2 cell lines

\section{Introduction}

Nowadays, chemotherapy, radiation, and surgery are classical treatment methods for cancer, but they have stark mental and biochemical side effects which predominantly destroy the healthy cells of patients. 5-FU is one of the most potent chemotherapeutic drugs which is efficient against several types of cancer such as skin cancer, breast cancer, pancreatic cancer, stomach cancer and is more effective in the curing of colorectal cancer [1,2]. 5-FU is a fluorinated pyrimidine antimetabolite converted within the cells into 5-fluorodeoxyuridine monophosphate which constrains the synthesis of DNA by inhibiting the cellular enzyme thymidylate synthetase leading to blocking the conversion of deoxyuridine acid to thymidine $[3,4]$.

The key challenge related to the oral administration of $5-\mathrm{FU}$ is the unconventional oral bioavailability due to which the intravenous administration of this drug is truly in clinical usage. However, intravenous administration leads to the quick distribution and elimination of 5-FU with an instant half-life of 8-20 minutes [5]. It was reported that 5-FU rapidly cleared from the plasma due to its rapid metabolism with a mean half-life 6.3 minutes and with regular peak plasma at $13.4 \mu \mathrm{g} / \mathrm{mL}$. Whereas oral administration of 5-FU had a normal peak plasma level of $8.3 \mu \mathrm{g} / \mathrm{ml}$ which happened $10-20$ minutes post-dosing with a short half-life of 7.2 minutes on account of the extreme first pass catabolism [6]. Moreover, dihydropyrimidine dehydrogenase enzyme which accountable to catabolism of 5-FU in the liver is furthermore found in high concentration in the gastrointestinal tract epithelium, while is not found in colon carcinomas, which enhance the susceptibility of colon cancers to 5-FU [7]. Also 5-FU can be PEGylated using short or long chain poly ethylene glycol, this idea depended on results obtained in our lab after PEGylation of octreotide [8]. Notwithstanding, the oral administration of 5-FU has a great variability in plasma levels which represent an important factor to oral as compared to administration intravenously as reported previously [9].

Conversely, intravenous administration of 5-FU yields severe systemic toxic effects of gastrointestinal (GIT), blood complaints, and skin diseases, due to the 5-FU cytotoxicity on healthful human cells. Hence, the need to formulate targeted delivery of 5-FU through oral route would not only diminish the exposure of healthy cells to the drug but also provide an efficient and harmless curative effect for colon cancer with a lower dose and reduced duration of therapy [5]. It was reported that the therapeutic effect of 5-FU can be augmented and its harmful effects can be suppressed by the precise accumulation of the anticancer agent in the tumor regions with continued exposure of the cells to this drug [10]. To extend the exposure time of cancer cells to 5-FU, the drug delivery was formulated by incorporation into microsponge formulation.

Microsponges (MS) are micro-sized particles with high porosity and unique ability for encapsulation of a wide multiplicity of pharmaceutical active ingredients. MS delivery system is used to target and modify the release of active ingredients from pharmaceutical formulations, enhance the stability and decrease the side effects [11]. MS have high load activity reach up to $90 \%$ entrapment efficiency [12], self-sterilizing

*Corresponding author: Ahmed AH Abdellatif, Department of Pharmaceutics and Industrial pharmacy, Faculty of Pharmacy, Al Azhar University, Assuit 71524, Egypt, Tel: +201027227142; E-mail: mahmoud.hassan.othman@gmail.com

Received September 19, 2016; Accepted January 05, 2017; Published January 09, 2017

Citation: Othman MH, Zayed GM, El-Sokkary GH, Ali UF, Abdellatif AAH (2017) Preparation and Evaluation of 5-Fluorouracil Loaded Microsponges for Treatment of Colon Cancer. J Cancer Sci Ther 9: 307-313. doi: 10.4172/1948-5956.1000433

Copyright: (c) 2017 Othman MH, et al. This is an open-access article distributed under the terms of the Creative Commons Attribution License, which permits unrestricted use, distribution, and reproduction in any medium, provided the original author and source are credited. 
with average pore size of $0.25 \mu \mathrm{m}$ where bacteria cannot penetrate [13] and they release the drugs in a sustained manner [13-15].

MS may efficiently be taken up by the macrophages in the colon, thus showed effective localized drug action at the site of action [5]. These characteristics make MS more advantageous over many drug delivery systems such as microcapsules and liposomes [14-16]. Moreover, targeting can be improved by altering the physicochemical properties such as size of the formulated NPs [17-21] or by attaching targeting ligand in vitro as well as in vivo [22-26]. Furthermore, the formulated NPs need to be identified using different analytical methods which are a novel approach for cancer targeting [20,25,27]. It was reported that a dualsensitive core/shell hybrid nanospheres, consisting of gold nanorods (GNR) as the core and poly ( $\mathrm{N}$-isopropyl acrylamide-co-methacrylic acid) as the shell were synthesized. The resulted nanospheres revealed significant applications of in controlled release of anticancer agents and have a synergistic end product of chem-photothermal therapy [27]. Silver and quantum dots hybrids microspheres were developed as a novel near-infrared photoluminescence (NIR PL) which showed highly selective and sensitive NIR PL responses to CA125 antigen, over other potential interferences $[28,29]$

This manuscript studied and developed new formula novel 5-FU loaded microsponges by Oil in Oil emulsion solvent diffusion method. These 5-FU loaded microsponges can be incorporated in specific formulations as colon targeting for treatment of colon cancer. These prepared 5-FU loaded microsponges are more effective than 5-FU on the cell lines. Our study depended on the formulation of 5-FU as MS in the form of Oil/Oil emulsion method to enhance the entrapping efficiency of more than $95 \%$.

The purpose of the present study was to prepare Eudragit RS 100MS containing 5-FU using an emulsion solvent diffusion method for colon cancer treatment. The study investigated the effect of $\mathrm{D}: \mathrm{P}$ ratio, magnesium stearate: solvent ratio and solvent: polymer ratio on the physicochemical characteristics of the prepared MS. The formulated MS were characterized for the particle sizes, entrapment efficiency and in vitro release. By comparing the in vitro release rate of 5-FU from MS, the best formula to be incorporated in colon-specific formulations has been selected for colon cancer targeting. Furthermore, the formulated MS were incubated with cell lines (HCT16 and CACO2) for determination of the internalization of 5-FU and for studying the toxicity of the formulations on the cell lines of colon cancer.

\section{Materials and Methods}

\section{Materials}

5-Fluorouracil was purchased from, Applichem for pharmaceuticals co., (Gmbh, Germany). Eudragit RS 100, phosphate buffer saline (PBS) MTT stain and dimethyl sulfoxide (DMSO) were purchased from Sigma Chemical Co. (St. Louis, USA). Light liquid paraffin and Magnesium stearate were purchased from El-Gomhoria Chem. Co. (Cairo, Egypt). Acetone, hydrochloric acid, orthophosphoric acid potassium dihydrogen phosphate, n-hexane and disodium hydrogen phosphate, were purchased from El-Nasr Chemical Co. (Cairo, (Egypt). Fetal bovine serum (FBS), DMEM media, and penicillin/streptomycin $(\mathrm{P} \backslash \mathrm{S})$ were purchased from Gibco (USA). Human colorectal cancer cells CACO-2 and human colon carcinoma cells HCT116 were purchased from VACSERA CO., Dukki, Egypt. The refined water utilized for all experiments was obtained using a distilled water purification system from double steam distillation device (Gesellschaft für $\mathrm{GmbH}$, Germany). All glasswares were thoroughly washed with freshly prepared aqua regia, extensively rinsed with water several times and oven-dried at $50^{\circ} \mathrm{C}$ before use.

\section{Preparation of Microsponges}

5-FU-MS were prepared using oil in oil emulsion solvent diffusion technique [12]. Eudragit RS 100 was dissolved in acetone, once a clear solution was obtained, $500 \mathrm{mg}$ of 5 -FU was added in addition to magnesium stearate ( $3 \% \mathrm{w} / \mathrm{v}$ solvent) and the whole mixture was kept in the ultrasonic bath adjusted at a $90-\mathrm{kHz}$ frequency for 5 minutes to obtain homogenous dispersion as shown in Table 1 . Then the mixture was poured into $150 \mathrm{ml}$ of liquid paraffin formerly cooled to $10^{\circ} \mathrm{C} \pm$ $0.5^{\circ} \mathrm{C}$ while the use of mechanical stirrer for 45 minutes. The oil in oil emulsion formed was progressively warmed to $35^{\circ} \mathrm{C} \pm 5^{\circ} \mathrm{C}$ and was stirred at this temperature for another 30 minutes. During this period of time, the acetone was entirely removed by diffusion into liquid paraffin and evaporated through the air/liquid interface. The solidified MS were purified, washed five times with $60 \mathrm{ml}$ of n-hexane, dried at room temperature for 12 hours and stored in a des iccator for further investigations (Figure 1).

\section{Variation of preparation parameters}

Many parameters were taken into consideration such as drugpolymer ratio, polymer-solvent ratio, and magnesium stearate-solvent ratio. Three D:P ratios, 1:1, 1:2, and 1:3 were investigated in the preparations F1, F2, and F3, respectively. Two other concentrations of magnesium stearate were studied, $1.5 \% \mathrm{w} / \mathrm{v}$ used in formulations $\mathrm{F} 4$, F6 and 5\% w/v in F5 and F7. The polymer/solvent ratio initially used in the preparation of MS was 1:5. However, this ratio was altered to 1:10 in formulations F8, F10 and 1:20 in formulations F9, F11, (Table 1).

\section{Determination of mean particle size}

The particle size studies were carried out using laser light scattering technique (Horiba LA-300 Light Scattering Particle Size Distribution Analyzer, Kyoto, Japan). The aqueous microsponges' suspension was first put into the sample chamber equipped with an ultrasonic probe to aid in the dispersion of cohesive particles. The MS was continuously re-circulated from the sample chamber via the sample flow cell. The achieved uniform dispersion was examined to measure particle size distribution. Particle size distribution was analyzed using the inbuilt software. Each sample was measured at three times and the average particle size was calculated as mean diameter $[13,16,17]$.

\section{Determination of encapsulation efficiency (EE)}

The EE percent was determined by accurate weighing a quantity of

\begin{tabular}{|c|c|c|c|c|c|}
\hline $\begin{array}{c}\text { Preparations } \\
\mathbf{( F )}\end{array}$ & $\begin{array}{c}\text { Eudragit RS } \\
\mathbf{1 0 0}(\mathbf{g})\end{array}$ & $\begin{array}{c}\text { Acetone } \\
\mathbf{( m l )}\end{array}$ & $\begin{array}{c}\text { DIP } \\
\text { ratio }\end{array}$ & $\begin{array}{c}\text { PIS } \\
\text { ratio }\end{array}$ & $\begin{array}{c}\text { \% wlv Mg } \\
\text { stearate } \\
\text { ratio }\end{array}$ \\
\hline F1 & 0.5 & 2.5 & $01: 01$ & $01: 05$ & 3 \\
\hline F2 & 1 & 5 & $01: 02$ & $01: 05$ & 3 \\
\hline F3 & 1.5 & 7.5 & $01: 03$ & $01: 05$ & 3 \\
\hline F4 & 0.5 & 2.5 & $01: 01$ & $01: 05$ & 1.5 \\
\hline F5 & 0.5 & 2.5 & $01: 01$ & $01: 05$ & 5 \\
\hline F6 & 1 & 5 & $01: 02$ & $01: 05$ & 1.5 \\
\hline F7 & 1 & 5 & $01: 02$ & $01: 05$ & 5 \\
\hline F8 & 0.5 & 5 & $01: 01$ & $01: 10$ & 3 \\
\hline F9 & 0.5 & 10 & $01: 01$ & $01: 20$ & 3 \\
\hline F10 & 1 & 10 & $01: 02$ & $01: 10$ & 3 \\
\hline F11 & 1 & 20 & $01: 02$ & $01: 20$ & 3 \\
\hline
\end{tabular}

Table 1: The composition of different MS prepared by $\mathrm{O} / \mathrm{O}$ emulsion solvent diffusion method. 


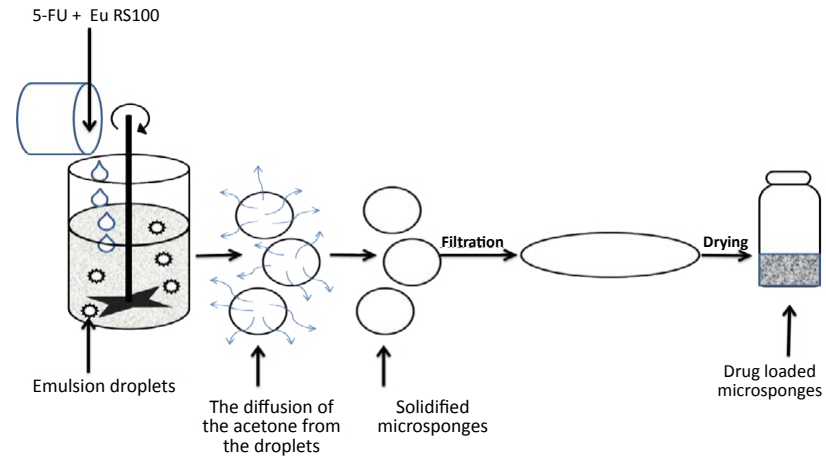

Figure 1: Graphical illustration of 5-FU loaded MS prepared by oil in oil solvent diffusion method.

MS equivalent to $10 \mathrm{mg}$ of 5-FU. Drug concentration was determined spectrophotometrically using (UV-1601 Shimadzu Co. Kyoto, Japan) [30]. 5-FU is sparingly soluble in water $(12 \mathrm{mg} / \mathrm{ml})$, so $10 \mathrm{mg}$ of drug (5-FU) equivalent of microsponges was crushed carefully in a glass mortar and transferred to a $100 \mathrm{ml}$ volumetric flask using phosphate buffer $\mathrm{pH}$ 6.8. The volumetric flask was completed to the volume with phosphate buffer $\mathrm{pH} 6.8$ then agitated in ultrasonic bath for 1 hour. The sample was filtered and the drug concentration was determined spectrophotometerically at $267 \mathrm{~nm}$ using phosphate buffer $\mathrm{pH} 6.8$ as a blank. The concentration was calculated using the standard calibration curve of 5-FU in phosphate buffer $\mathrm{pH} 6.8$ and $\mathrm{EE} \%$ was calculated using the following equation:

$$
\mathrm{EE} \%=\frac{\text { Actual amount of drug }}{\text { Theoretical amount of drug }} \times \text { i } 00
$$

Eudragit RS 100 and magnesium stearate are water insoluble so these is no interference of the excipients.

\section{Infrared Fourier transform spectroscopy analysis (FTIR)}

5-FU, Eudragit RS 100, 5-FU-Eudragit RS100 physical mixture and optimized formula (F3) were subjected to infrared (IR) analysis, the samples were scanned in the range from $400 \mathrm{~cm}^{-1}$ to $4000 \mathrm{~cm}^{-1}$ and recorded on an FTIR (SSP-10 A Shimadzu Co., Japan), using the $\mathrm{KBr}$ disk technique [30].

\section{Scanning electron microscopy}

Scanning electron microscope (SEM) was used for investigation of the shape and surface morphology of MS. The MS were fixed with carbon-Glue and covered regularly with gold palladium within argon atmosphere. Samples were then investigated with a JEM-100 S, (Japan), scanning electron microscope [12].

\section{In vitro drug release study}

USP type II dissolution test apparatus (Paddle) was used for in vitro dissolution studies of 5-FU-MS. $50 \mathrm{mg}$ of the 5-FU equivalent of MS was placed in $900 \mathrm{ml}$ phosphate buffer ( $\mathrm{pH} 6.8)$ stirred at $50 \mathrm{rpm}$ and the temperature was kept at $37^{\circ} \mathrm{C} \pm 0.5^{\circ} \mathrm{C}$ [31]. At predetermined time interval, $5 \mathrm{ml}$ samples were pulled out and substituted with an equal volume of fresh buffer. The concentration in the pulled out samples was determined spectrophotometrically at $267 \mathrm{~nm}$. Each sample was implemented in three times and expressed as mean values \pm standard deviation.

\section{Kinetic data}

The dissolution profile of each formulation was fitted to various models such as zero order kinetics, first order kinetics, Higuchi, and Korsmeyer-Peppas to assess the kinetics of drug release from prepared 5-FU-MS [32].

\section{Cell culture}

Human colorectal cancer cells CACO-2 and human colon carcinoma cells HCT116 were maintained in DMEM cell culture media containing $10 \%$ FBS and $1 \%$ penicillin/streptomycin. Cells were grown at $37^{\circ} \mathrm{C}$ in a $5 \% \mathrm{CO}_{2}$ humidified atmosphere. The effect of the F2, F3, and 5-FU on cell viability was investigated using MTT cell viability assay [33]. The principles of MTT assay are based on the cleavage of the insoluble tetrazolium salt to a detectable colored formazan product by succinate-tetrazolium reductase enzyme; that exists in the mitochondria and it is only active in the viable cells. The produced insoluble formazan is directly proportional to the activity of the mitochondrial dehydrogenase enzyme and consequently to the portion of the viable cells. The intracellular formazan dye resulting from the metabolically active cells can be solubilized and quantified by measuring its absorbance using ELISA reader [34]. Briefly, the cells were cultivated in 96-well plates $\left(5 \times 10^{3} /\right.$ well $)$ in $100 \mu$ of DMEM media. On the following day, the experimental media F2, F3, and 5-FU were added and then incubated for 24 hours. After addition of fifty $\mu \mathrm{l}$ of MTT solution ( $2 \mathrm{mg} / \mathrm{ml}$ in PBS), every well was incubated for 4 hours. Each well were received $150 \mu \mathrm{l}$ of DMSO, after, careful removal of the media, and then after shaking the absorbance was measured at $570 \mathrm{~nm}$ using an ELISA reader (Molecular Devices, Sunnyvale, USA).

\section{Results and Discussion}

Spherical, uniform, regular and free flowing MS were successfully prepared by oil in oil emulsion solvent diffusion method. Investigations proved that, when the drug to polymer ratio was increased, the entrapment efficiency was increased due to decrease the total drug lost at the steps of the fabrication process. The fact that more amount of drug was present per unit polymer so F1 (1:1, drug to polymer ratio) has higher drug content than F3 (1:3, drug to polymer ratio). The production yield was very high due to the existence of the nonpolar liquid paraffin in the extrinsic phase which inhibits the escape of ingredients used in the formulation of MS [35].

Magnesium stearate was used as stabilizer to overcome the problem of coalescence flocculation of particles during solvent diffusion by the reduction of the phase tension between the formed microsponges and liquid paraffin [36,37]. The influence of $\mathrm{Mg}$ stearate concentration $(1.5,3$ and $5 \% \mathrm{w} / \mathrm{v})$ on the characteristics of the prepared MS showed, that low concentration $(1.5 \% \mathrm{w} / \mathrm{v})$ produced aggregated MS. The microsponges obtained by using medium concentration of magnesium stearate $(3 \% \mathrm{w} / \mathrm{v})$ were spherical in shape, uniform, regular and free flowing. However, increasing magnesium stearate concentration to $5 \%$ led to neutralization the electric charge of Eudragit RS-100 due to assembling of magnesium stearate on the surface of MS that produced aggregation of MS [38]. It has been reported that magnesium stearate reduced the interfacial tension and prevented the electrical charges and flocculation during the preparation of microsponges in general [12]. The variation of the magnesium stearate concentration $(1.5,3$ and $5 \%)$ in the formulations did not have any influence on the release profiles of the drug.

The decrease in polymer/solvent ratio resulted in a significant decrease in the particle size of prepared MS. This observation was 
imputed to the fact which when the dispersed phase with higher viscosity poured into the dispersion medium of high viscosity (liquid paraffin), the emulsion was barely broken into small droplets, and the larger ones were formed. It was reported that the particle size of microparticles was proportional to the dispersed phase viscosities. In such a way, increasing the dispersed phase viscosity resulted in increasing the particle size of microparticles (Table 2) [26-28]. 5-FU were prepared previously by another group as MS [39], using the technique W/O/W emulsification method. This method showed a good formulation but with lower\% EE, which showed maximum $\% \mathrm{EE}$ of about $59 \%$. This is due to the aqueous solubility of 5-FU, 5-FU has a certain solubility of around $12 \mathrm{mg} / \mathrm{ml}$ water, these in turn make the loaded 5-FU microsponges prepared oil in water emulsion contained very low drug content as 5-FU escaped to aqueous external phase. Our study showed that the formulation of 5-FU as MS in the form of oil/oil emulsion method has a higher\% EE of more than $95 \%$, this is due to the insolubility of 5-FU in external phase, this make our formula has a better\% EE than the other one.

The results showed that the increase in polymer concentration resulted in increasing the particle size of MS. When acetone diffuses out, almost the dispersed phase is turned into solid MS and separated particles appear. Therefore, in high drug to polymer ratios, less polymer amounts surrounded the drug and decreased the particle sizes of MS. When the dispersed phase with elevated viscosity teemed into the dispersion medium bigger droplets were formed and mean particle sizes increased [40]

\section{Infrared Fourier transform spectroscopy analysis (FTIR)}

FT-IR spectroscopy is an indication of the functional groups in the scanned samples either of the polymers or drug. Since IR spectroscopy measures the sample's absorbance spectrums rapidly and directly with a controllable sampling depth and with little or no preparation of the sample [30-34]. For this reason, FT-IR was applied to confirm the compatibility of all used additives with 5-FU. FTIR spectrum of 5-FU showed a distinctive peak appear in $3000 \mathrm{~cm}^{-1}$ to $2900 \mathrm{~cm}^{-1}$; represents $\mathrm{C}-\mathrm{H}$ stretching, bands in the region $1429 \mathrm{~cm}^{-1}$ to $1660 \mathrm{~cm}^{-1}$ corresponding to the $\mathrm{C}=\mathrm{C}$ and $\mathrm{C}=\mathrm{N}$ ring vibrations. The peaks at about $1348 \mathrm{~cm}^{-1}$ due to pyrimidine compound vibration. The peaks at 1180 $\mathrm{cm}^{-1}$ and $1246 \mathrm{~cm}^{-1}$ corresponded to the $\mathrm{C}-\mathrm{O}$ and $\mathrm{C}-\mathrm{N}$ vibrations, respectively [41]. Eudragit RS 100 showed a $\mathrm{C}=\mathrm{O}$ stretching band around $1726 \mathrm{~cm}^{-1}$ [42]. The FTIR scanning of the physical mixture of 5-FU with Eudragit RS 100 and the selected formula (F3) showed the characteristic fingerprint of both drug and polymer. These observations explained that there is no chemical interaction or changes took place during the mixing formulation of MS with added materials (Figure 2).

\begin{tabular}{|c|c|c|c|}
\hline $\begin{array}{c}\text { Formula } \\
\text { No. }\end{array}$ & $\begin{array}{c}\text { \% Production Yield } \\
\mathbf{\pm} \text { SD }\end{array}$ & $\%$ EE \pm SD & $\begin{array}{c}\text { Mean particle size } \\
(\boldsymbol{\mu m}) \pm \text { SD }(\mathbf{n m})\end{array}$ \\
\hline F1 & $87.20 \pm 2.10$ & $95.44 \pm 2.67$ & $67.05 \pm 24.74$ \\
\hline F2 & $87.70 \pm 3.22$ & $91.18 \pm 0.19$ & $92.57 \pm 29.06$ \\
\hline F3 & $92.21 \pm 1.78$ & $88.24 \pm 1.22$ & $118.12 \pm 48.21$ \\
\hline F4 & $76.50 \pm 2.26$ & $101.90 \pm 2.60$ & $80.50 \pm 36.08$ \\
\hline F5 & $62.76 \pm 1.06$ & $97.90 \pm 2.98$ & $68.00 \pm 39.85$ \\
\hline F6 & $82.47 \pm 1.80$ & $93.00 \pm 3.07$ & $103.10 \pm 29.95$ \\
\hline F7 & $89.80 \pm 2.00$ & $90.76 \pm 1.05$ & $91.80 \pm 32.75$ \\
\hline F8 & $90.00 \pm 3.01$ & $90.90 \pm 4.74$ & $60.40 \pm 38.33$ \\
\hline F9 & $87.10 \pm 2.14$ & $77.80 \pm 0.19$ & $53.11 \pm 41.03$ \\
\hline F10 & $91.50 \pm 0.94$ & $74.80 \pm 2.67$ & $89.80 \pm 30.09$ \\
\hline F11 & $93.80 \pm 1.75$ & $74.58 \pm 1.62$ & $85.40 \pm 34.00$ \\
\hline
\end{tabular}

Table 2: Production yield, percentage recovery (drug content) of different Microsponges and mean particle size.

\section{Scanning electron microscopy (SEM)}

The scanning electron images of the selected MS (F3) was observed by SEM analysis that, the MS were finely spherical and uniform (Figure 3a). More detailed image indicated several pores on the MS surfaces (Figure $3 \mathrm{~b}$ ). The numerous pores present in microsponges surface were induced by the diffusion of the solvent from the emulsion droplets during the formation of the MS [43]. These pores enable the dissolution medium to penetrate the into MS to dissolve and release the entrapped drug from microsponges [44].

\section{In vitro release study}

In vitro release of pure 5-FU and different 5-FU-MS is illustrated in Figure 4. The release of 5-FU from MS was characterized by an immediate burst release followed by a moderately slow release. The initial rapid release is attributed to the dissolution of drug adsorbed in microsponge's surfaces. The later relatively slow release rate could be attributed to release and diffusion of the drug from the interior of MS. The drug release was characterized by biphasic pattern matrix diffusion kinetics [45]. Primarily, diffusion of the aqueous dissolution medium into pores of MS results in dissolution of the drug. After that, diffusion of the drug through the microsponges pores into the dissolution medium [44]. The result obtained from Figure 4 showed that the in vitro release of 5-FU from the prepared MS was retarded with increasing the polymer/solvent ratio. This due to faster precipitation of the polymer around the drug in higher polymer/solvent concentrations. The fast polymer precipitation due to rapid acetone evaporation led to higher drug content and decreases the diffusion of the drug in the external phase identical results were gained by Kondo et al. [46]. The low drug release rate could be also attributed to the decrease in a number of pores at MS surface at a higher polymer to drug ratios or an increase of internal phase viscosity [47]. The delay in drug release also could be imputed to an increase in the diffusional path length that the drug has to overpass.

\section{Kinetic analysis of release profiles}

The release patterns were fitted on various kinetic models such zero, first, Higuchi and Korsmeyer-Peppas. The correlation coefficient of the straight line that has the highest value was used to compare between different models. Table 3 listed the cor-relation coefficients (r) of all formulated MS. The obtained data revealed that the release profiles of formulae from F1 to F9 follow Higuchi diffusion model while release profiles of formulae of F10 and F11 follow Peppas diffusion model. The diffusion mechanism of drug release was due to the presence of porous structure on the surface of MS which enables easy penetration of the release media that dissolves the 5-FU and it diffuses through the diffusion paths (pores).

\section{Cellular toxicity}

$\mathrm{IC}_{50}$ values are very dependent on the stipulations under which they are measured. Generally, the concentration of inhibitor is higher; the more drug activity will be lowered. $\mathrm{IC}_{50}$ value increases as 5-FU concentration increases. Furthermore depending on the type of inhibition other agents may affect $\mathrm{IC}_{50}$ value; for ATP-dependent enzymes, $\mathrm{IC}_{50}$ value has an interdependency with the concentration of ATP. IC $_{50}$ values were used for comparison of the potency between two drugs [48]. The $\mathrm{IC}_{50}$ of a drug can be determined by building up a doseresponse curve and inspecting the effect of different concentrations of the formula on contrary 5-FU activity. $\mathrm{IC}_{50}$ values can be calculated for a certain new formula by measuring the concentration that needs to inhibition of half of the maximum biological response of the 5-FU [48] 

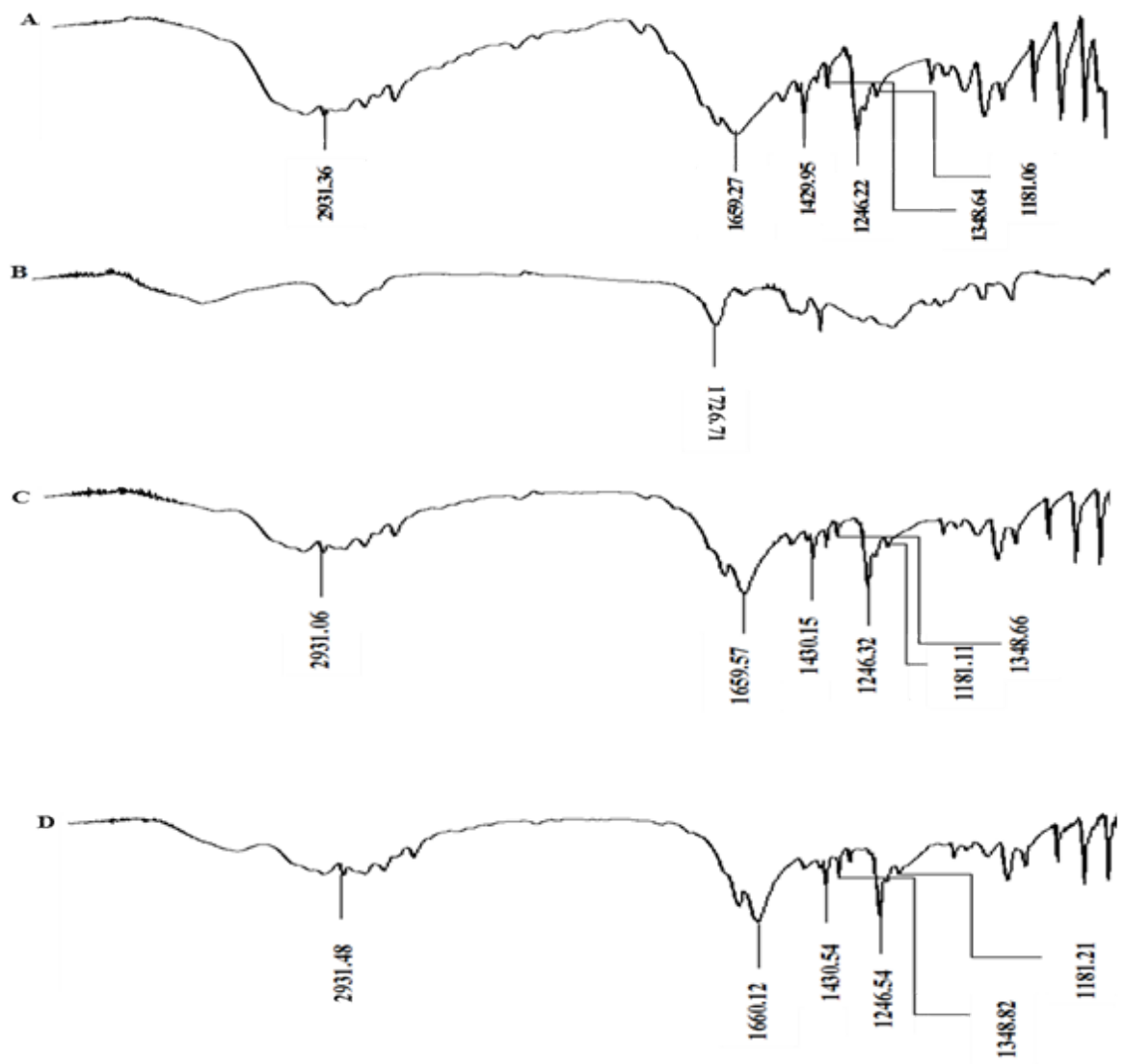

Figure 2: FTIR spectroscopy of (A) 5-FU, (B) Eudragit RS 100, (C) 5-FU_Eudragit RS 100 in physical mixture and (D) F3 (1:3, drug to polymer ratio).

A)
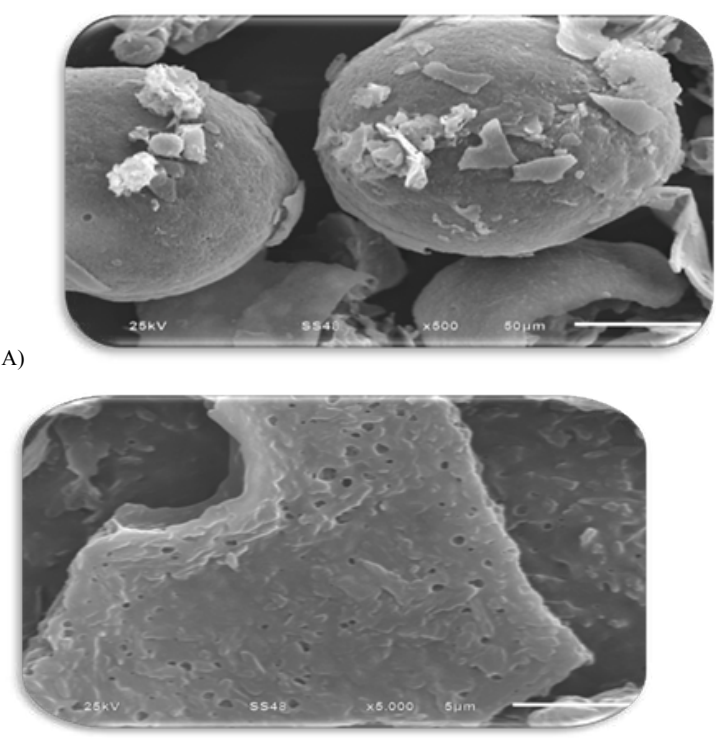

B)

Figure 3: Scanning electron micrographs of 5-Fluorouracil-Eudragit RS 100 microsponges (F3), a) the whole microsponges, b) high magnification power of microsponges showed the characteristic pores.

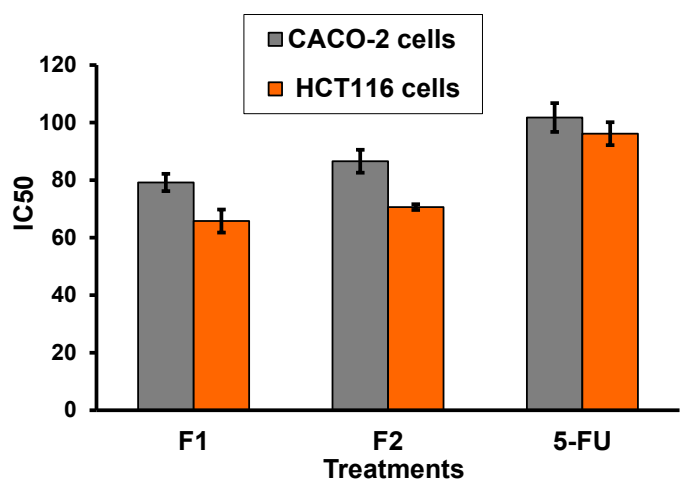

Figure 4: $\mathrm{IC}_{50}$ of different formulae on CACO2 and HCT116 cell lines.

The effect of formulations F3 and F2 compared to the effect of the drug (5-FU) on human colorectal cancer cells CACO-2 and HCT116 was investigated by MTT assay and the results (Figure 5). The cell viability experiment was designed as a tool for evaluation the efficacy of the different concentrations of drug samples used in the current research. The control sample was already used to calculate the $\mathrm{IC}_{50}$ but not included in the histogram, as this control cell showed no data. For more confirmation the experiments were repeated 5 times. MTT 
Citation: Othman MH, Zayed GM, El-Sokkary GH, Ali UF, Abdellatif AAH (2017) Preparation and Evaluation of 5-Fluorouracil Loaded Microsponges for Treatment of Colon Cancer. J Cancer Sci Ther 9: 307-313. doi: 10.4172/1948-5956.1000433

\begin{tabular}{|c|c|c|c|c|c|c|c|c|c|c|c|}
\hline \multirow{2}{*}{ Correlation Coefficient } & \multicolumn{11}{|c|}{ Formula No. } \\
\hline & F1 & F2 & F3 & F4 & F5 & F6 & F7 & F8 & F9 & F10 & F11 \\
\hline Zero & 0.71 & 0.8 & 0.9 & 0.567 & 0.656 & 0.878 & 0.87 & 0.743 & 0.771 & 0.713 & 0.832 \\
\hline First & 0.93 & 1 & 1 & 0.859 & 0.957 & 0.988 & 0.99 & 0.97 & 0.97 & 0.944 & 0.965 \\
\hline $\begin{array}{l}\text { Higushi } \\
\text { diffusion }\end{array}$ & 0.94 & 1 & 1 & 0.929 & 0.97 & 0.99 & 0.99 & 0.984 & 0.987 & 0.938 & 0.955 \\
\hline Korsemey-er-Peppas & 0.94 & 0.9 & 0.9 & 0.923 & 0.949 & 0.975 & 0.97 & 0.952 & 0.957 & 0.959 & 0.985 \\
\hline Order & \multicolumn{9}{|c|}{ Diffusion model } & \multicolumn{2}{|c|}{ Kors.-Pepp } \\
\hline
\end{tabular}

Table 3: The kinetics parameter correlation coefficient ( $r$ ) of zero, first, Higuchi and Peppas models for different microsponges formulations.

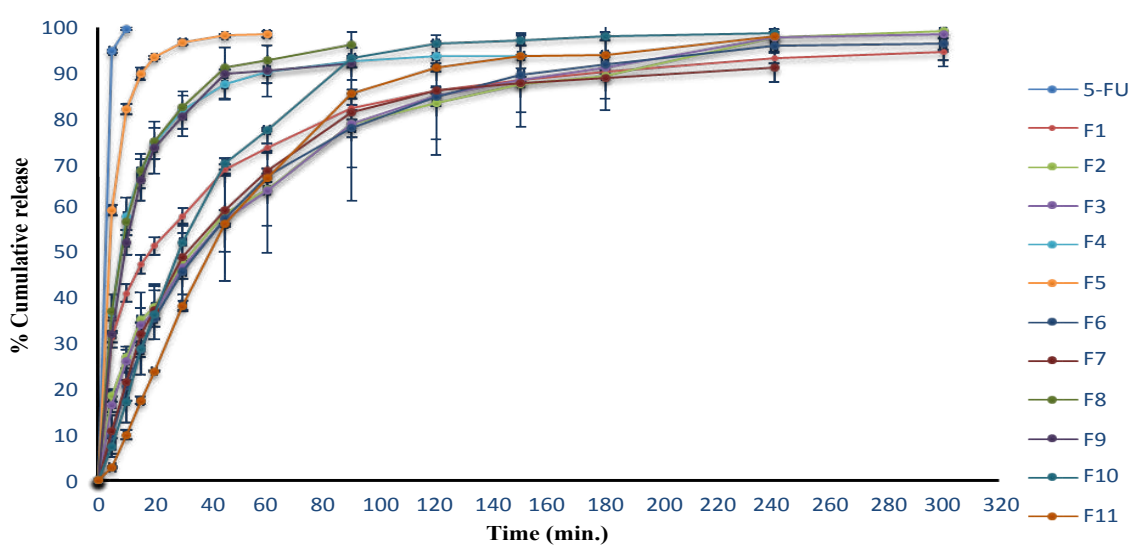

Figure 5: In vitro release of 5-Fluorouracil from Eudragit RS 100 microsponges

measure the cell viability and as you know the non-viable cells are died, so the two are the same. In CACO-2 cell line, F3 was effective than F2 and drug itself. Quantitatively, $\mathrm{IC}_{50}$ was 79.2, 86.6 and $101.2 \mathrm{ng} /$ $\mu \mathrm{l}$ for formulations F3, F2, and 5-FU, respectively. At the same time, in HCT116 cell line the $\mathrm{IC}_{50}$ was $65.8,70.6$ and $96.2 \mathrm{ng} / \mu \mathrm{l}$ for F3, F2, and 5-FU, respectively indicate that $\mathrm{F} 3$ still the most effective formula. Comparing between the two cell lines (CACO-2 and HCT116) the given formulae F3, F2, and 5-FU exerted more toxicity on HCT116 versus those of CACO-2.

Our results showed that $\mathrm{F} 3$ was the most toxic formula on both cell lines (CACO-2 and HCT116) as indicated by less $\mathrm{IC}_{50}$.

\section{Conclusion}

From the previous results, it was possible to conclude that oil in oil solvent diffusion represented a successful method of encapsulation of 5-FU with relatively high encapsulation efficiencies. The addition of magnesium stearate is essential for the successful formulation of MS. The release profiles showed that all formulations obey the diffusion mechanisms, F3 was more effective on colorectal cell lines than F2 and 5-FU.

\section{Future prospectus}

On account of 5-FU is the first drug of choice in the treatment of colon cancer, our plan in the future is to formulate the tablet having MS containing the 5-FU as a colon specific delivery system in a specific oral dosage form used for colon targeting for treatment of colon cancer.

\section{References}

1. Sutar PS, Joshi V (2013) Preparation and characterisation of 5-Fluorouracil loaded PLGA Nanoparticles for Colorectal Cancer Therapy. Unique J pharm Biol sci 1(2): Page 52-58.

2. Arias $\mathrm{JL}$ (2008) Novel strategies to improve the anticancer action of 5-fluorouracil by using drug delivery systems. Molecules 13: 2340-2369.

3. Sweetman SC (2011) Martindale the complete drug reference, London.

4. Parker WB, Cheng YC (1990) Metabolism and mechanism of action of 5-fluorouracil. Pharmacol Ther 48: 381-395.

5. Gupta A, Tiwari G, Tiwari R, Srivastava R (2015) Factorial designed 5 -fluorouracil-loaded microsponges and calcium pectinate beads plugged in hydroxypropyl methylcellulose capsules for colorectal cancer. Int J Pharm Investig 5: 234.

6. Phillips TA, Howell A, Grieve RJ, Welling PG (1980) Pharmacokinetics of oral and intravenous fluorouracil in humans. J pharm sci 69: 1428-1431.

7. Myers CE (1976) Pharmacokinetics of the fluoropyrimidines: Implications for their clinical use. Cancer treat rev 3: 175-183.

8. Abdellatif AA, Osman SK, Ali MTM, Darras FH (2005) N-terminal monoPEGylation of octreotide. Pharm Res 22: 743-749.

9. Bruckner HW, Creasey WA (1974) The administration of 5-fluorouracil by mouth. Cancer 33: 14-18.

10. Arias JL, Ruiz MA, López-Viota M, Delgado AV (2008) Poly (Alkyl cyanoacrylate) colloidal particles as vehicles for anti-tumour drug delivery: a comparative study. Colloids and Surf B: Biointerfaces 62: 64-70.

11. Jelvehgari M (2006) The microsponge delivery system of benzoyl peroxide: Preparation, characterization and release studies. Int J Pharm 308: 124-132.

12. Zaki Rizkalla CM, latif Aziz R, Soliman II (2011) In vitro and in vivo evaluation of hydroxyzine hydrochloride microsponges for topical delivery. AAPS PharmSciTech 12: 989-1001.

13. Shaha V (2010) Microsponge drug delivery: A Review. Int J Res Pharm Sci 1 212-218. 
Citation: Othman MH, Zayed GM, El-Sokkary GH, Ali UF, Abdellatif AAH (2017) Preparation and Evaluation of 5-Fluorouracil Loaded Microsponges for Treatment of Colon Cancer. J Cancer Sci Ther 9: 307-313. doi: 10.4172/1948-5956.1000433

14. Abdellatif A (2016) A novel topical spray formulation for Ginkgo Bioloba for Antifungal Activity. J Nanomed Nanotechnol 7: 2

15. Hamd MAE, Abdellatif AAH, Derayea SM, Abdelmageed OH, Askal HF (2015) Spectrophotometric determination of Nifedipine and Nicardipine in their pharmaceutical preparations. Ind Chem 1: 103

16. Abdellatif AA, Tawfeek TM (2015) Transfersomal nanoparticles for enhanced transdermal delivery of Clindamycin. AAPS PharmSciTech 17: 1067-1074.

17. Abdellatif AA, Abou-Taleb HA (2014) Transfersomal nanoparticles of keratolytic and antibacterial agents for enhanced transdermal delivery. J Nanotechol Adv Mater 4: 19-23.

18. Abdellatif AAH, El Hamd MA, Saleh KI (2016) A formulation, optimization and evaluation of controlled released alginate beads Loaded-Flurbiprofen. J Nanomed Nanotechnol 7:357.

19. Abdellatif A, Tawfeek HM (2016) Metformin loaded Carbopol gel for lowering the intra-abdominal visceral fat. J Bioequiv Availab 8: 149-152.

20. Abdellatif AA, Abou-Taleb HA (2015) Optimization of nano-emulsion formulations for certain emollient effect.

21. Abdellatif A, Tawfeek HM (2016) Erratum to: Transfersomal nanoparticles for enhanced transdermal delivery of Clindamycin. AAPS PharmSciTech.

22. Cheng M, Gao X, Wang, Y, Chen H, He B, et al. (2013) Synthesis of liver-targeting dual-ligand modified GCGA/5-FU nanoparticles and their characteristics in vitro and in vivo. Int J Nanomedicine 8: 4265-4276.

23. Phatak P, Cookson JC, Dai F, Smith V, Gartenhaus RB, et al. (2007) Telomere uncapping by the G-quadruplex ligand RHPS4 inhibits clonogenic tumour cell growth in vitro and in vivo consistent with a cancer stem cell targeting mechanism. Br J Cancer 96: 1223-1233.

24. Abdellatif AAH (2015) Targeting of somatostatin receptors using quantum dots nanoparticles decorated with Octreotide. J Nanomed Nanotechnol S6:005

25. Abdellatif AA, El Rasoul SA, Osman S (2015) Gold nanoparticles decorated with octreotide for somatostatin receptors targeting. Int J Pharm Sci Res 7 : $14-20$.

26. Abdellatif AA (2016) Novel gold nanoparticles coated with somatostatin as a potential delivery system for targeting somatostatin receptors. Drug Dev Ind Pharm 42: 1782-1791.

27. Jin H (2015) Facile synthesis of gold nanorods/hydrogels core/shell nanospheres for $\mathrm{pH}$ and near-infrared-light induced release of 5-fluorouracil and chemo-photothermal therapy. Colloids Surf B Biointerfaces 128: 498-505.

28. Jin H, Gui R, Gong J, Huang W (2016) Aptamer and 5-fluorouracil dual-loading $\mathrm{Ag} 2 \mathrm{~S}$ quantum dots used as a sensitive label-free probe for near-infrared photoluminescence turn-on detection of CA125 antigen. Biosensors and Bioelectronics.

29. Hassan WAA (2011) Formulation and Evaluation of Drug loaded Microspheres for Potential Application, in Pharmaceutics, Al-Azhar University: Cairo 44-45.

30. Pandav S, Naik J (2014) Preparation and in vitro evaluation of ethylcellulose and polymethacrylate resins loaded microparticles containing hydrophilic drug. J Pharm 2014: 1-5

31. Srivastava R, Kumar D, Pathak K (2015) Colonic luminal surface retention of meloxicam microsponges delivered by erosion based colon-targeted matrix tablet. Int J pharm 427: 153-162.

32. Jain V, Singh R (2010) Dicyclomine-loaded Eudragit $®-$ based microsponge with potential for colonic delivery: preparation and characterization. Trop J Pharm Res 9 .

33. Mosmann T (1983) Rapid colorimetric assay for cellular growth and survival: application to proliferation and cytotoxicity assays. J Immunol Methods 65: 55-63.

34. Ismail IA, Kang KS, Lee HA, Kim JW, Sohn YK (2007) Genistein-induced neuronal apoptosis and G2/M cell cycle arrest is associated with MDC1 upregulation and PLK1 down-regulation. Eur J Pharmacol 575: 12-20.

35. Sandor M, Enscore D, Weston P, Mathiowitz E (2001) Effect of protein molecular weight on release from micron-sized PLGA microspheres. J Control Release 76: 297-311.

36. Goto S, Kawata M, Nakamura M, Aoyama T (1985) Effect of magnesium stearate in preparation of eudragit RS-microcapsules containing drugs. Yakugaku zasshi: J Pharm Soci Japan 105: 1087-1095.

37. Arshady R (1991) Preparation of biodegradable microspheres and microcapsules: 2 Polyactides and related polyesters. J Control Release 17: 1-21.

38. Chong-Kook K, Mi-Jung K, Kyoung-Hee O (1994) Preparation and evaluation of sustained release microspheres of terbutaline sulfate. Int J pharm 106: 213-219.

39. Gupta A, Tiwari G, Tiwari R, Srivastava R (2015) Factorial designed 5 -fluorouracil-loaded microsponges and calcium pectinate beads plugged in hydroxypropyl methylcellulose capsules for colorectal cancer. Int J Pharm Investig 5: 234-246.

40. Nokhodchi A, Jelveghari M, Siahi MR, Dastmalchi S (2005) The effect of formulation type on the release of benzoyl peroxide from microsponges. Iranian J Pharm Sci 1: 131-142.

41. Yassin AEB, Khalid Anwer MD, Hammam AM, Ibrahim ME, Mohsen AB (2010) Optimization of 5-flurouracil solid-lipid nanoparticles: A preliminary study to treat colon cancer. Int J Med Sci 7: 398.

42. Jain V, Singh R (2010) Development and characterization of Eudragit RS 100 loaded microsponges and its colonic delivery using natural polysaccharides. Acta Pol Pharm 67: 407-415.

43. Crotts G, Park Tk (1995) Preparation of porous and nonporous biodegradable polymeric hollow microspheres. J Control Release 35: 91-105.

44. Freiberg S, Zhu X (2004) Polymer microspheres for controlled drug release. Int $\mathrm{J}$ pharm 282: 1-18

45. Lemoine $D(1998)$ Preparation and characterization of alginate microspheres containing a model antigen. Int J pharm 176: 9-19.

46. Kondo N, Iwao T, Hirai K, Fukuda M, Yamanouchi K, et al. (1994) Improved oral absorption of enteric coprecipitates of a poorly soluble drug. $\mathrm{J}$ pharm sci 83: $566-570$

47. Omaima AS, Mohammed AH, Nagia AM, Ahmed SZ (2016) Formulation and optimization of mouth dissolve tablets containing Rofecoxib solid dispersion. AAPS PharmSciTech 7: 167-175.

48. NIH, Assay Operations for SAR Support, N.I.o. Health, Editor, National Institute of Health. 